\title{
Magnetic Schrödinger operators with discrete spectra on non-compact Kähler manifolds
}

\author{
Nicolae Anghel
}

\begin{abstract}
We identify a class of magnetic Schrödinger operators on Kähler manifolds which exhibit pure point spectrum. To this end we embed the Schrödinger problem into a Dirac-type problem via a parallel spinor and use a Bochner-Weitzenböck argument to prove our spectral discreteness criterion.
\end{abstract}

\section{Introduction}

Let $(M, g)$ be a complete non-compact oriented Riemannian manifold of dimension $n \geq 2$, with Riemannian metric $g$, and let $a$ be a real 1 -form on $M$, of class $C^{\infty}$. Then $a$ induces a metric connection $\nabla^{a}$ on the trivial Hermitian bundle $M \times \mathbf{C}$, identifiable to the first order differential operator

$$
C^{\infty}(M, \mathbf{C}) \ni \phi \longmapsto d^{a} \phi:=d \phi+i \phi a \in C^{\infty}\left(M, T^{*} M \otimes \mathbf{C}\right),
$$

where $d$ represents ordinary exterior differentiation and $i=\sqrt{-1}$. As usual, the Riemannian metric allows one to consider pointwise Hermitian products $\langle\cdot, \cdot\rangle_{x}, x \in M$, in the complexified cotangent bundle $T^{*} M \otimes \mathbf{C}$ and, via the volume form, global (integrated) Hermitian products $(\cdot, \cdot)$, in the spaces $C_{\mathrm{cpt}}^{\infty}(M, \mathbf{C})$ and $C_{\mathrm{cpt}}^{\infty}\left(M, \mathbf{C} \otimes T^{*} M\right)$. With respect to these products the formal adjoint $\left(d^{a}\right)^{*}$ of $d^{a}$ can be defined as a first order differential operator,

$$
\left(d^{a}\right)^{*}: C^{\infty}\left(M, \mathbf{C} \otimes T^{*} M\right) \longrightarrow C^{\infty}(M, \mathbf{C}),
$$

Key Words: Magnetic Schrödinger operator, Magnetic field, Discrete spectrum, Dirac operator, Kähler manifold.

2010 Mathematics Subject Classification: Primary: 35J10, 58J50. Secondary: 35P05, 47F05, 53C55, 81V10.

Received: August, 2011.

Accepted: February, 2012. 
and then the magnetic Schrödinger operator (magnetic bottle) with magnetic potential $a$ is the second order differential operator $H_{a}:=\left(d^{a}\right)^{*} d^{a}$, viewed as an unbounded operator in $L^{2}(M, \mathbf{C})$. (see Section 2 for more details). It is known that regardless of $a, H_{a}$ with domain $C_{\mathrm{cpt}}^{\infty}(M, \mathbf{C})$ is an essentially self-adjoint operator in $L^{2}(M, \mathbf{C})[\mathrm{S} 1]$.

There is a great deal of work, especially on Euclidean spaces $M=\mathbf{R}^{n}$, dedicated to deciding which magnetic Schrödinger operators $H_{a}$ have discrete spectrum, that is a spectrum consisting only in isolated eigenvalues of finite multiplicity [AHS, I, KS, A1]. Typically, these works provide sufficient conditions for spectral discreteness, in terms of the magnetic field $B$ associated to $a, B:=d a$.

The purpose of this note is to provide one more result along these lines, in the case $M$ is a Kähler manifold with Kähler form $\omega$ and Riemannian metric $g$ naturally induced by $\omega$. This result can easily be seen to generalize that of [A1], when $n$ is even.

Theorem. Let $M$ be a non-compact Kähler manifold with Kähler form $\omega$ and Riemannian metric induced by $\omega$. Assume that $H_{a}$ is a magnetic Schrödinger operator on $M$ associated to a real 1-form a of class $C^{\infty}$. Then $H_{a}$ has discrete spectrum if the real-valued function $\langle B(x), \omega(x)\rangle$ on $M$, where $\langle\cdot, \cdot\rangle$ denotes the natural pointwise inner product on 2-forms, satisfies the condition

$$
\lim _{x \rightarrow \infty}\langle B(x), \omega(x)\rangle=-\infty .
$$

\section{Magnetic Schrödinger operators on manifolds}

Let $(M, g)$ be a complete non-compact oriented Riemannian $\left(C^{\infty}\right)$ manifold of dimension $n$, equipped with the metric $g$. On the usual real $C^{\infty}$-bundles of $p$-forms on $M, \Lambda^{p}\left(T^{*} M\right), 0 \leq p \leq n$, consider the standard inner products $\langle\cdot, \cdot\rangle_{x}, x \in M$. Specifically, if $\left(e_{1}, e_{2}, \ldots, e_{n}\right)$ is an oriented local orthonormal frame in the tangent bundle $T M$, with local dual frame of 1-forms in the cotangent bundle $T^{*} M,\left(e_{1}^{*}, e_{2}^{*}, \ldots, e_{n}^{*}\right)$, then a local orthonormal basis of $\Lambda^{p}\left(T^{*} M\right)$ is $\left\{e_{J}^{*}\right\}_{J}, e_{J}^{*}:=e_{j_{1}}^{*} \wedge e_{j_{2}}^{*} \wedge \cdots \wedge e_{j_{p}}^{*}$, where $J$ runs through the set of all multi-indices $1 \leq j_{1}<j_{2}<\cdots<j_{p} \leq n$.

There is a Levi-Cività metric connection $\nabla^{\mathrm{LC}}$ on $\Lambda^{p}\left(T^{*} M\right)$, extending naturally the Levi-Cività connection on $T^{*} M$, the exterior product connection; For a local vector field $e$ in $T M$ and local forms $v^{*}$ in $T^{*} M$ and $\phi$ in $\Lambda^{p}\left(T^{*} M\right)$,

$$
\nabla_{e}^{\mathrm{LC}}\left(v^{*} \wedge \phi\right)=\nabla_{e}^{\mathrm{LC}} v^{*} \wedge \phi+v^{*} \wedge \nabla_{e}^{\mathrm{LC}} \phi .
$$

Denote now by $\Omega^{p}(M, \mathbf{C}):=C^{\infty}\left(M, \Lambda^{p}\left(T^{*} M\right) \otimes \mathbf{C}\right)$ the Hermitian vector space of $C^{\infty}$ complex global $p$-forms and by

$$
d: \Omega^{p}(M, \mathbf{C}) \longrightarrow \Omega^{p+1}(M, \mathbf{C})
$$


the usual exterior differential. In terms of the complexified Levi-Cività metric connection $\nabla^{\mathrm{LC}}$ on $\Lambda^{p}\left(T^{*} M\right) \otimes \mathbf{C}, d$ can be written locally as

$$
d=\sum_{j=1}^{n} e_{j}^{*} \wedge \nabla_{e_{j}}^{\mathrm{LC}}
$$

Fix now $a \in \Omega^{1}(M, \mathbf{R})$ a real global 1-form. Then the twisted differential $d^{a}:=d+i a \wedge$, defined on $\Omega^{p}(M, \mathbf{C})$ by

$$
\Omega^{p}(M, \mathbf{C}) \ni \phi \longmapsto d^{a} \phi=d \phi+i a \wedge \phi \in \Omega^{p+1}(M, \mathbf{C}),
$$

has the local frame counterpart

$$
d^{a}=\sum_{j=1}^{n} e_{j}^{*} \wedge \nabla_{e_{j}}^{\mathrm{LC}, a}
$$

where $\nabla^{\mathrm{LC}, a}$ is the twisted metric connection on $\Lambda^{p}\left(T^{*} M\right) \otimes \mathbf{C}$ defined by

$$
\nabla_{v}^{\mathrm{LC}, a} \phi=\nabla_{v}^{\mathrm{LC}} \phi+i a(v) \phi, \quad v \text { global vector field in } T M, \phi \in \Omega^{p}(M, \mathbf{C}) . \quad(3)
$$

For $\phi \in \Omega^{p}(M, \mathbf{C})$ and $\psi \in \Omega_{\mathrm{cpt}}^{p}(M, \mathbf{C})$ the global Hermitian product $(\phi, \psi):=$ $\int_{M}\langle\phi, \psi\rangle d v o l$ induces the formal adjoint $\left(d^{a}\right)^{*}$ of $d^{a}$,

$$
\left(d^{a}\right)^{*}: \Omega^{p+1}(M, \mathbf{C}) \longrightarrow \Omega^{p}(M, \mathbf{C}),
$$

subject to

$$
\left(\left(d^{a}\right)^{*} \phi, \psi\right)=\left(\phi, d^{a} \psi\right), \quad \phi \in \Omega^{p+1}(M, \mathbf{C}), \psi \in \Omega_{\mathrm{cpt}}^{p}(M, \mathbf{C}) .
$$

It follows that locally

$$
\left.\left(d^{a}\right)^{*}=-\sum_{j=1}^{n} e_{j}\right\lrcorner \nabla_{e_{j}}^{\mathrm{LC}, a},
$$

where $\left.e_{j}\right\lrcorner$ denotes interior multiplication (contraction) by the local vector field $e_{j}$

Making in the above discussion $p=0$ we get a second order differential operator

$$
H_{a}:=\left(d^{a}\right)^{*} d^{a}: C^{\infty}(M, \mathbf{C}) \longrightarrow C^{\infty}(M, \mathbf{C}) .
$$

Seen as an unbounded operator in $L^{2}(M, \mathbf{C})$, the completion of $C_{\mathrm{cpt}}^{\infty}(M, \mathbf{C})$ with respect to $(\cdot, \cdot), H_{a}$ is called the (scalar) magnetic Schrödinger operator generated by the potential $a$. It is then a nice exercise to see that in a local frame,

$$
H_{a}=-\sum_{j=1}^{n}\left(e_{j}+i a\left(e_{j}\right)\right)^{2}+\sum_{j=1}^{n}\left(\nabla_{e_{j}}^{L C} e_{j}+i a\left(\nabla_{e_{j}}^{L C} e_{j}\right)\right)
$$


$H_{a}$ with domain $C_{\mathrm{cpt}}^{\infty}(M, \mathbf{C})$ can be closed in only one way in $L^{2}(M, \mathbf{C})$, i.e., $H_{a}$ is an essentially self-adjoint operator [S1].

In this note we will be interested in reasonably simple conditions on $M$ and $a$ which would ensure that $H_{a}$ has pure point spectrum. We therefore conclude this section with a general criterion for spectral discreteness.

Proposition 1. $H_{a}$ being defined as above, if there is a function $f \in C^{0}(M, \mathbf{R})$, $\lim _{x \rightarrow \infty} f(x)=\infty$, such that

$$
\left(H_{a} \phi, \phi\right) \geq(f \phi, \phi), \quad \phi \in C_{c p t}^{\infty}(M, \mathbf{C}),
$$

then $H_{a}$ has discrete spectrum.

Proof. We will supply a somewhat less traditional proof to this proposition. To this end, let $W^{2}(M, a)$ be the domain of the unique closed extension of $H_{a}$ from $C_{\mathrm{cpt}}^{\infty}(M, \mathbf{C})$ into $L^{2}(M, \mathbf{C}) . W^{2}(M, a)$ is the completion of $C_{\mathrm{cpt}}^{\infty}(M, \mathbf{C})$ with respect to the Sobolev inner product $(\cdot, \cdot)_{2}:=(\cdot, \cdot)+\left(H_{a} \cdot H_{a} \cdot\right)$. Since $H_{a}: W^{2}(M, a) \longrightarrow L^{2}(M, \mathbf{C})$ is self-adjoint, its spectrum is contained in the real line.

To prove the proposition it suffices to show that for every $\lambda \in \mathbf{R}$ the operator $H_{a}-\lambda$ with domain $W^{2}(M, a)$ is Fredholm, since for any Fredholm operator 0 is an isolated point of its spectrum, and in fact an eigenvalue with finite multiplicity.

Fix now a number $\lambda \in \mathbf{R}$. The assumption on the function $f$ provides a compact subset $K$ of $M$ such that $f(x) \geq \lambda+1$, if $x \in M \backslash K$. The hypothesis (4) and the density of $C_{\mathrm{cpt}}^{\infty}(M, \mathbf{C})$ in $W^{2}(M, a)$ imply that

$$
\left(\left(H_{a}-\lambda\right) \phi, \phi\right)-((f-\lambda) \phi, \phi)_{K} \geq(\phi, \phi)_{M \backslash K}, \quad \phi \in W^{2}(M, a),
$$

where for a subset $U$ of $M,(\cdot, \cdot)_{U}$ indicates integration is carried out only over $U$.

As in [A2], $H_{a}-\lambda$ will be a Fredholm operator if we can show that any sequence $\left\{\phi_{n}\right\}_{n}$ from $W^{2}(M, a)$, which is $L^{2}$-bounded and for which $\left\{\left(H_{a}-\right.\right.$ ג) $\left.\phi_{n}\right\}_{n}$ is $L^{2}$-convergent, admits a $L^{2}$-convergent subsequence.

Since $\left\{\phi_{n}\right\}_{n}$ is bounded in the Sobolev norm $\|\cdot\|_{2}$, by Rellich's lemma [S2] the sequence $\left\{\left.\phi_{n}\right|_{K}\right\}_{n}$ has a convergent subsequence in $L^{2}(K, \mathbf{C})$ (assumed to be the sequence itself).

The property (5) applied now to the differences $\left\{\phi_{m}-\phi_{n}\right\}_{m, n}$ shows that $\left\{\left.\phi_{n}\right|_{M \backslash K}\right\}_{n}$ is a Cauchy sequence in $L^{2}(M \backslash K, \mathbf{C})$. We conclude that $\left\{\phi_{n}\right\}_{n}$ converges in the $L^{2}$-norm, since its restrictions to $K$ and $M \backslash K$ do so. 


\section{Generalized Dirac operators}

As mentioned in the introduction, our spectral discreteness analysis will come about by embedding the magnetic Schrödinger operator formalism into a Dirac-type framework. It is then desirable to briefly review here the concept of generalized Dirac bundle with its associated Dirac operator [GL].

If $(M, g)$ is, as before, a complete non-compact oriented Riemannian manifold of dimension $n$, let $C l(M)$ be the real Clifford bundle of algebras induced by the tangent bundle $T M$ and the Riemannian metric $g$. There is a canonical embedding $T M \subset C l(M)$, and then the Riemannian metric and Levi-Cività connection extend from $T M$ to $C l(M)$ in such a way that the connection $\nabla^{L C}$ of $C l(M)$ preserves the metric and acts as a derivation.

A complex bundle of left modules over the bundle of algebras $C l(M)$, say $S \longrightarrow M$, will be called a (generalized) Dirac bundle if $S$ is furnished with a Hermitian metric $\langle\cdot, \cdot\rangle$ and a metric connection $\nabla^{S}$ such that

i) The action on $S$ by unit vectors in $T M \subset C l(M)$ is a pointwise isometry.

ii) The connection $\nabla^{S}$ is compatible with the Clifford multiplication, in the sense that for local sections $e$ in $T M, \phi$ in $C l(M)$, and $s$ in $S$, we have

$$
\nabla_{e}^{S}(\phi \cdot s)=\left(\nabla_{e}^{L C} \phi\right) \cdot s+\phi \cdot\left(\nabla_{e}^{S} s\right) .
$$

Above, the "." indicates the action of $C l(M)$ on $S$, while the multiplication in $C l(M)$ will be simply represented by juxtaposition. Since $T M$ generates $C l(M)$, the action - of $C l(M)$ on $S$ is completely determined by its restriction to $T M$.

There are several fundamental examples and constructs of Dirac bundles associated to $M$, which are relevant to us:

a) $S=C l(M) \otimes \mathbf{C}$. In this case $C l(M)$ acts on $S$ by left algebra multiplication and $\nabla^{S}$ is the complexification of $\nabla^{L C}$.

b) $S=\Lambda\left(T^{*} M\right) \otimes \mathbf{C}$. This case, where $\Lambda\left(T^{*} M\right)$ represents the real bundle of exterior forms on $M$, is relevant to our concept of magnetic Schrödinger operator, in the sense that the scalar concept we work with admits an extension to a concept of exterior form magnetic Schrödinger operator.

If $\left(e_{1}, e_{2}, \ldots, e_{n}\right)$ is a local frame in $T M$ then the action - of $e_{j}$ on $S$ is given by $\left.e_{j} \cdot=e_{j}^{*} \wedge-e_{j}\right\lrcorner . \nabla^{S}$ is the exterior form extension of the LeviCività connection $\nabla^{\mathrm{LC}}$ on $T^{*} M$, cf. (2). In fact case $b$ ) coincides with case $a$ ) under the canonical vector bundle linear isometry $\Lambda\left(T^{*} M\right) \simeq C l(M), e_{J}^{*} \longmapsto$ $e_{j_{1}} e_{j_{2}} \ldots e_{j_{p}}$. This is a vector bundle isomorphism which also preserves the Levi-Cività connections, but of course not an algebra bundle isomorphism.

c) For a Kähler manifold $M$ of complex dimension $m[\mathrm{GH}]$ let $\omega$ be the Kähler 2-form and let $g$ be the Riemannian metric naturally induced on $T M$ by $\omega$. Then the integrable complex structure $\mathrm{J}$ in the tangent bundle $T M$ makes 
$(T M, g)$ a Hermitian bundle, and there is a complex linear isometry between $(T M, \mathrm{~J})$ and the Hermitian bundle of $(0,1)$-forms $T^{* 0,1} M \subset T^{*} M \otimes \mathbf{C}$. Since $M$ is Kähler this isometry takes the Levi-Cività connection of $T M$ to the unique anti-holomorphic Hermitian connection $\nabla_{\bar{z}}$ on $T^{* 0,1} M$. Then $S:=$ $\Lambda\left(T^{* 0,1} M\right)$ is a Dirac bundle, when endowed with a Clifford multiplication similar to that of case $b$ ), via the above-said complex isometry, and with the exterior product connection induced by, and extending, $\nabla_{\bar{z}}[\mathrm{~B}]$.

d) If $M$ is a spin manifold [LM] then $\mathrm{S}$ can be taken to be the spinor bundle $\Sigma(M)$ of $M$. To be more specific, for a spin manifold the principal $\mathrm{SO}(n)$ bundle $P_{\mathrm{SO}}(M)$ of oriented frames in $T M$ lifts to a principal Spin-bundle $P_{\text {Spin }}(M)$, equivariantly with respect to the 2-cover map $\operatorname{Spin}(n) \longrightarrow \mathrm{SO}(n)$. The spinor bundle $\Sigma(M)$ is then the fiber product $\Sigma(M):=P_{\mathrm{Spin}}(M) \times_{\mu} \Delta$, where $\Delta$ is an irreducible representation of the Euclidean Clifford algebra on $n$ generators $C l_{n} \otimes \mathbf{C}$ and $\mu$ is the unitary representation $\mu: \operatorname{Spin}(n) \longrightarrow U(\Delta)$ induced by the left multiplication with elements of $\operatorname{Spin}(n) \subset C l_{n} \otimes \mathbf{C}$. We get then the compatible connection $\nabla^{\text {Spin }}$ of $\Sigma(M)$ by lifting the Riemannian connection on $P_{\mathrm{SO}}(M)$ to $P_{\mathrm{Spin}}(M)$, via the Lie algebra isomorphism $\operatorname{so}(n) \simeq$ $\operatorname{spin}(n)$.

e) If $S$ is a Dirac bundle and $F$ is any Hermitian bundle over $M$, equipped with a metric connection $\nabla^{F}$, then the twisted bundle $S \otimes F$ is naturally a Dirac bundle, with Clifford multiplication induced by that of $S$ and connection $\nabla^{S \otimes F}:=\nabla^{S} \otimes I d+I d \otimes \nabla^{E}$.

Any Dirac bundle $S$ generates a distinguished differential operator $D_{S}$ : $C^{\infty}(M, S) \longrightarrow C^{\infty}(M, S)$, the generalized Dirac operator, defined as follows: If $m: T^{*} M \otimes S \longrightarrow S$ denotes the restriction to $T^{*} M$ (metrically identified with $T M)$ of the Clifford action - of $C l(M)$ on $S$, then $D_{S}=m \circ \nabla^{S}$. Locally, $D_{S}$ admits the representation

$$
D_{S}=\sum_{j=1}^{n} e_{j} \cdot \nabla_{e_{j}}^{S}
$$

where as usual $\left(e_{1}, e_{2}, \ldots, e_{n}\right)$ is a local orthonormal frame in $T M$.

Since $M$ is complete, $D_{S}$ with domain $C_{\mathrm{cpt}}^{\infty}(M, S)$ is an essentially selfadjoint first order elliptic differential operator in $L^{2}(M, S)$ [GL].

Clearly, the Dirac operator associated to $S=\Lambda\left(T^{*} M\right) \otimes \mathbf{C}$ (case $b$ ) above) is $d+d^{*}$, where $d$ is the exterior differential and $d^{*}$ its formal adjoint, as in section 2 .

In case $c$ ), when $M$ is a Kähler manifold and $S=\Lambda\left(T^{* 0,1} M\right)$ the Dirac operator becomes $\sqrt{2}\left(\bar{\partial}+\bar{\partial}^{*}\right)$, where $\bar{\partial}$ is the Dolbeault operator and $\bar{\partial}^{*}$ its formal adjoint $[\mathrm{B}]$.

On a spin manifold $M$ the Dirac operator associated to the spinor bundle $\Sigma(M)$ of case $d)$ is called the classical Dirac operator. 
For the square of a generalized Dirac operator $D_{S}$ the following BochnerWitzenböck formula holds true [GL],

$$
D_{S}^{2}=\left(\nabla^{S}\right)^{*} \nabla^{S}+\mathcal{R}^{S},
$$

where $\mathcal{R}^{S}$ is the Hermitian curvature bundle morphism acting on $S$ according to the formula

$$
\mathcal{R}^{S}=\sum_{j<k} e_{j} \cdot e_{k} \cdot R_{e_{j}, e_{k}}^{S}, \quad R_{e_{j}, e_{k}}^{S}=\left[\nabla_{e_{j}}^{S}, \nabla_{e_{k}}^{S}\right]-\nabla_{\left[e_{j}, e_{k}\right]}^{S} .
$$

In case $b), \mathcal{R}^{\Lambda\left(T^{*} M\right) \otimes \mathbf{C}}$ preserves $\Lambda^{p}\left(T^{*} M\right) \otimes \mathbf{C}$ and evidently, $\left.\mathcal{R}^{\Lambda\left(T^{*} M\right) \otimes \mathbf{C}}\right|_{\Lambda^{0}\left(T^{*} M\right) \otimes \mathbf{C}}=0$.

In case $d), \mathcal{R}^{\Sigma(M)}=k / 4$, where $k$ is the scalar curvature of the spin manifold $M$ (Lichnerowicz's theorem [LM]).

In case $e), \mathcal{R}^{S \otimes F}$ can be written as

$$
\mathcal{R}^{S \otimes F}=\mathcal{R}^{S} \otimes I d+\sum_{j<k} e_{j} \cdot e_{k} \cdot \otimes R_{e_{j}, e_{k}}^{F} .
$$

If $F=\mathbf{C}_{a}$, the trivial bundle $M \times \mathbf{C}$ equipped with the metric connection $\nabla^{a}$ associated to some real 1-form $a \in \Omega^{1}(M, \mathbf{R})$, as in the introduction, then $S \otimes \mathbf{C}_{a}=S$, and so (6) becomes $\mathcal{R}^{S \otimes \mathbf{C}_{a}}=\mathcal{R}^{S}+i \rho^{a}$., where $\rho^{a}$ is the global section of $C l(M)$ given by

$$
\rho^{a}=\sum_{j<k} R_{e_{j}, e_{k}}^{a} e_{j} e_{k}, \quad R_{e_{j}, e_{k}}^{a}=e_{j}\left(a\left(e_{k}\right)\right)-e_{k}\left(a\left(e_{j}\right)\right)-a\left(\left[e_{j}, e_{k}\right]\right) .
$$

It is elementary to see that under the linear isometry $\Lambda\left(T^{*} M\right) \simeq C l(M)$ explained at case $b$ ) above, $\rho^{a} \in C^{\infty}(M, C l(M))$ is the image of the real 2-form $B=d a \in \Omega^{2}(M, \mathbf{R})$.

Finally, if $S=\Lambda\left(T^{*} M\right) \otimes \mathbf{C}$ and $F=\mathbf{C}_{a}$, then $\nabla^{\left(\Lambda\left(T^{*} M\right) \otimes \mathbf{C}\right) \otimes \mathbf{C}_{a}}=\nabla^{\mathrm{LC}, a}$, in the notation of section 2 , cf. (3). The connection Laplacian $\left(\nabla^{\mathrm{LC}, a}\right)^{*} \nabla^{\mathrm{LC}, a}$ can then be called an exterior form magnetic Schrödinger operator, since it restricts to $H_{a}$ on $\Omega^{0}(M, \mathbf{C})$.

\section{Our results}

We are now ready to state and prove an abstract discreteness criterion for certain $H_{a}$ 's and, as an application, supply a proof to the theorem given in the introduction.

Proposition 2. Suppose that are given a non-compact Riemannian manifold $(M, g)$, a real 1-form $a \in \Omega^{1}(M, \mathbf{R})$ with associated scalar Schrödinger operator $H_{a}$, and a generalized Dirac bundle $S$ over $M$ with Clifford multiplication $\cdot$, compatible connection $\nabla^{S}$, and Dirac operator $D_{S}$. 
In addition, suppose that there exists a $\nabla^{S}$-parallel global section $\sigma \in$ $C^{\infty}(M, S)$ such that

$$
\lim _{x \rightarrow \infty}\left\langle i \rho^{a} \cdot \sigma, \sigma\right\rangle=-\infty,
$$

where $\rho^{a}$ is the global section of $C l(M)$ given by (7). Then the magnetic Schrödinger operator $H_{a}$ has discrete spectrum.

Proof. Consider the twisted Dirac bundle $S \otimes \mathbf{C}_{a}$ and its Dirac operator $D_{S \otimes \mathbf{C}_{a}}$. We have the Bochner-Weitzenböck formula

$$
D_{S \otimes \mathbf{C}_{a}}^{2}=\left(\nabla^{S \otimes \mathbf{C}_{a}}\right)^{*} \nabla^{S \otimes \mathbf{C}_{a}}+\mathcal{R}^{S}+i \rho^{a} \cdot
$$

which will be applied to sections of type $\phi \sigma=\sigma \otimes \phi \in C_{\mathrm{cpt}}^{\infty}\left(M, S \otimes \mathbf{C}_{a}\right)$, for arbitrary $\phi \in C_{\mathrm{cpt}}^{\infty}(M, \mathbf{C})$.

Therefore,

$\left(D_{S \otimes \mathbf{C}_{a}}^{2}(\phi \sigma), \phi \sigma\right)=\left(\nabla^{S \otimes \mathbf{C}_{a}} \sigma \otimes \phi, \nabla^{S \otimes \mathbf{C}_{a}} \sigma \otimes \phi\right)+\left(\phi \mathcal{R}^{S} \sigma, \rho \sigma\right)+\left(i \phi \rho^{a} \cdot \sigma, \phi \sigma\right)$.

However, $\nabla^{S \otimes \mathbf{C}_{a}} \sigma \otimes \phi=\nabla^{S} \sigma \otimes \phi+\sigma \otimes d^{a} \phi=\sigma \otimes d^{a} \phi$, since $\sigma$ is $\nabla^{S}$-parallel. For the same reason, $\mathcal{R}^{S} \sigma=0$. By the hypothesis (8), $\sigma$ is non-trivial, and since $\nabla^{S}$ is a metric connection, $\langle\sigma, \sigma\rangle$ is a (positive) constant function on $M$. By scaling $\sigma$ appropriately we can assume that $\langle\sigma, \sigma\rangle=1$.

Consequently, $\left(\nabla^{S \otimes \mathbf{C}_{a}} \sigma \otimes \phi, \nabla^{S \otimes \mathbf{C}_{a}} \sigma \otimes \phi\right)=\left(\sigma \otimes d^{a} \phi, \sigma \otimes d^{a} \phi\right)=$ $\int_{M}\langle\sigma, \sigma\rangle\left\langle d^{a} \phi, d^{a} \phi\right\rangle d v o l=\int_{M}\left\langle d^{a} \phi, d^{a} \phi\right\rangle d v o l=\left(H_{a} \phi, \phi\right)$.

Equation (9) now becomes

$$
\left\|D_{S \otimes \mathbf{C}_{a}}(\phi \sigma)\right\|^{2}=\left(H_{a} \phi, \phi\right)+\left(\left\langle i \rho^{a} \cdot \sigma, \sigma\right\rangle \phi, \phi\right),
$$

which implies

$$
\left(H_{a} \phi, \phi\right) \geq\left(-\left\langle i \rho^{a} \cdot \sigma, \sigma\right\rangle \phi, \phi\right) .
$$

The result follows by applying Proposition 1 to the function $f=-i\left\langle\rho^{a}\right.$. $\sigma, \sigma\rangle$, in the presence of the hypothesis (8).

A successful application of the above proposition rests obviously on the ability of finding Dirac bundles with non-trivial parallel sections $\sigma$ for which $\left\langle\rho^{a} \cdot \sigma, \sigma\right\rangle$ can be effectively computed. This is indeed the case with the theorem stated in the introduction.

Proof of the Theorem. For a Kähler manifold of complex dimension $m, n=$ $2 m$. If $\omega$ is the Kähler form inducing the Riemannian metric $g$ and if $\mathrm{J}$ is the integrable complex structure on $T M$ then there is a local orthonormal frame $\left(e_{1}, \mathrm{~J} e_{1}, e_{2}, \mathrm{~J} e_{2}, \ldots, e_{m}, \mathrm{~J} e_{m}\right)$ in $T M$ such that $\omega=e_{1}^{*} \wedge\left(\mathrm{J} e_{1}\right)^{*}+e_{2}^{*} \wedge\left(\mathrm{J}_{2}\right)^{*}+$ $\cdots+e_{m}^{*} \wedge\left(\mathrm{J} e_{m}\right)^{*}$. Expanding on the discussion on Kähler manifolds initiated in 
section 3, case $c), T^{* 0,1} M$ is the space dual to $T^{0,1} M:=\left\{v \in T^{*} M \otimes \mathbf{C} \mid \mathrm{J} v=\right.$ $-i v\}$. Since a local orthonormal basis of $T^{0,1} M$ is $\left\{\bar{\epsilon}_{1}, \bar{\epsilon}_{2}, \ldots, \bar{\epsilon}_{m}\right\}, \bar{\epsilon}_{j}:=$ $\frac{1}{\sqrt{2}}\left(e_{j}+i \mathrm{~J} e_{j}\right)$, a local orthonormal basis of $T^{* 0,1} M$ will be $\left\{\bar{\epsilon}_{1}{ }^{*}, \bar{\epsilon}_{2}{ }^{*}, \ldots, \bar{\epsilon}_{m}{ }^{*}\right\}$, with $\bar{\epsilon}_{j}{ }^{*}:=\frac{1}{\sqrt{2}}\left(e_{j}^{*}-i\left(\mathrm{~J} e_{j}\right)^{*}\right)$. So, for the Dirac bundle $\Lambda\left(T^{* 0,1} M\right)$ a local orthonormal basis for $\Lambda^{p}\left(T^{* 0,1} M\right)$ is $\left\{\bar{\epsilon}_{J}{ }^{*}\right\}_{J}, \bar{\epsilon}_{J}{ }^{*}=\bar{\epsilon}_{j_{1}}{ }^{*} \wedge \bar{\epsilon}_{j_{2}}{ }^{*} \wedge \ldots \bar{\epsilon}_{j_{p}}{ }^{*}, J=$ $\left(j_{1}, j_{2}, \ldots, j_{p}\right) p$-multi-index.

The Clifford multiplication in $\Lambda\left(T^{* 0,1} M\right)$ is then implemented by setting

$$
\left.\left.e_{j} \cdot=\bar{\epsilon}_{j}^{*} \wedge-\bar{\epsilon}_{j}\right\lrcorner, \quad\left(\mathrm{~J} e_{j}\right) \cdot=i\left(\bar{\epsilon}_{j}^{*} \wedge+\bar{\epsilon}_{j}\right\lrcorner\right), \quad j=1,2, \ldots, m .
$$

In preparation for applying proposition 2 notice that $\sigma:=1 \epsilon$ $C^{\infty}\left(M, \Lambda^{0}\left(T^{* 0,1} M\right)\right)$ is a parallel section of $\left.\Lambda\left(T^{* 0,1} M\right)\right)$. An elementary calculation based on (10) and (7) shows now that

$$
\left\langle i \rho^{a} \cdot \sigma, \sigma\right\rangle=\sum_{j=1}^{m} R_{e_{j}, \mathrm{~J} e_{j}}^{a} .
$$

The theorem follows from proposition 2 and the hypothesis (1), since $a=$ $\sum_{j=1}^{m} a\left(e_{j}\right) e_{j}^{*}+\sum_{j=1}^{m} a\left(\mathrm{~J} e_{j}\right)\left(\mathrm{J} e_{j}\right)^{*}$ implies $\langle d a, \omega\rangle=\sum_{j=1}^{m} R_{e_{j}, \mathrm{~J} e_{j}}^{a}=\left\langle i \rho^{a} \cdot \sigma, \sigma\right\rangle$.

Acknowledgment. Part of this work was completed while the author visited the Simion Stoilow Mathematical Institute of the Romanian Academy (IMAR) on a Bitdefender Professorship. The author would like to thank IMAR for hospitality and support.

\section{References}

[A1] N. Anghel, On a Class of Magnetic Schrödinger Operators with Discrete Spectrum, Proc. Amer. Math. Soc. 140, No. 5, 1613-1616, (2012).

[A2] N. Anghel, An Abstract Index Theorem on Non-Compact Riemannian Manifolds, Houston J. Math. 19, 223-237, (1993).

[AHS] J. Avron, I. Herbst, B. Simon, Schrödinger Operators with Magnetic Fields, I. General Interactions, Duke Math. J. 45, 847-883, (1978).

[B] J-M. Bismut, Demailly's Asymptotic Morse Inequalities. A Heat Equation Proof, J. Funct. Anal. 72, 263-278, (1987).

[D] A. Dufresnoy, Un Example de Champ Magnétique dans $\mathbf{R}^{\nu}$, Duke Math. J. 50, 729-734, (1983). 
[GH] P. Griffiths, J. Harris, Principles of Algebraic Geometry, John Wiley \& Sons, New York, (1978).

[GL] M. Gromov, B. Lawson, Positive Scalar Curvature and the Dirac Operator on Complete Riemannian Manifolds, Publ. Math. IHES, 58, 295-408, (1983).

[HM] B. Helffer, A. Mohamed, Caractérisation du Spectre Essentiel de l'Opérateur de Schrödinger avec un Champ Magnétique, Ann. Inst. Fourier 38, 95-112, (1988).

[I] A. Iwatsuka, Magnetic Schrödinger Operators with Compact Resolvent, J. Math. Kyoto Univ. 26, 357-374, (1986).

[KS] V. Kondratiev, M. Shubin, Discreteness of Spectrum for the Magnetic Schrödinger Operators, Commun. Partial Diff. Eqns 27, 477-525, (2002).

[LM] B. Lawson, M-L. Michelsohn, Spin Geometry, Princeton Univ. Press, Princeton, NJ, (1989).

[S1] M. Shubin, Essential Self-Adjointness for Magnetic Schrödinger Operators on Non-Compact Manifolds, Sémin. Équ. Dériv. Partielles, École Polytech., Palaiseau, (1999).

[S2] Pseudo-Differential Operators and Spectral Theory, Springer V., Berlin, (1974).

Nicolae ANGHEL,

Department of Mathematics,

University of North Texas,

Denton, TX 76203, USA.

Email: anghel@unt.edu 